\title{
THE SPECTRUM OF SASAKIAN MANIFOLDS
}

\author{
By YHUjI SHIBUYA
}

\section{$\S 0$. Introduction.}

The spectrum of a manifold, which is the set of eigenvalues of Laplacian, is in some sense related to the "pure tone" of the manifold.

There is an old question asking, "Can you hear the shape of the drum?", that is, to what extent can you determine some geometric character of the manifold by knowing its spectrum ? (See Kac [4])

In particular, we are interested in the question of whether a manifold is isometric to a sphere, if the spectrum of the manifold is the same as that of the sphere. This question has been affirmatively answered in the 1, 2, 3, 4, 5 and 6 dimensional cases. (See Berger, etc. [1] and Tanno [8]) But it is an open question for other dimensions.

In this paper, we affirmatively answer this question in the (5), 7, 9, 11 and 13 dimensional cases under the assumption that the manifold is a Sasakian manifold, which is a contact manifold with certain integrability conditions.

For the proof, we first establish several curvature properties of a Sasakian manifold, then study some geometric implications of the vanishing of the contact Bochoner curvature tensor, and finally we use the asymptotic expansion of the fundamental solution of the heat equation to express the spectral condition in terms of curvatures. The main theorem is then obtained for the wider class of spaces in which spheres are included.

\section{$\S 1$. Sasakian manifolds and their curvature properties.}

Let $M^{2 n+1}$ be a $2 n+1$-dimensional differentiable manifold. $M^{2 n+1}$ is said to have an almost contact structure if the structural group of its tangent bundle is reducible to $U(n) \times 1$, where $U(n)$ is an $(n, n)$ unitary group.

An almost contact structure can also be seen from a different point of view. A differentiable manifold $M^{2 n+1}$ is said to have a $(\varphi, \xi, \eta)$-structure if it admits an endomorphism $\varphi$ of the tangent spaces, a vector field $\xi$, and a 1 -form $\eta$ satisfying

$$
\eta(\xi)=1
$$

Received April 10, 1979

This research is partially supported by the graduate assistantship of Michigan State University. 
and

$$
\varphi^{2}=-I+\eta \otimes \xi,
$$

where $I$ denotes the identity transformation. It is easily seen that $\varphi$ satisfies

$$
\varphi \xi=0 \text { and } \eta \circ \varphi=0
$$

that is, $\varphi$ has rank $2 n$. It is easily proved that the notions of an almost contact structure and a $(\varphi, \xi, \eta)$-structure are equivalent. In this sense we sometimes refer to an almost contact structure $(\varphi, \xi, \eta)$.

We also see that $M^{2 n+1}$ admits a special Riemannian metric called a compatible metric such that

$$
g(\varphi X, \varphi Y)=g(X, Y)-\eta(X) r(Y) .
$$

$M^{2 n+1}$ with $(\varphi, \xi, \eta)$-structure and then metric (1.4) is said to have $(\varphi, \xi, \eta, g)$ structure or an almost contact metric structure $(\varphi, \xi, \eta, g)$.

The fundamental 2-form $\Phi$ of an almost contact metric structure $(\varphi, \xi, \eta, g)$ is defined by

$$
\Phi(X, Y)=g(\varphi X, Y) .
$$

$\Phi$ is skew-symmetric because of (1.2), (1.3) and (1.4).

An odd dimensional euclidean space $\boldsymbol{R}^{2 n+1}$, a hypersurface in an almost complex manifold, especially an odd dimensional sphere, a product manifold $M^{2 n} \times \boldsymbol{R}$ of an almost complex manifold and the real line, and a Brieskorn manifold are examples of almost contact metric manifolds.

An almost contact manifold $M^{2 n+1}$ is said to be normal if an almost complex structure of $M^{2 n+1} \times \boldsymbol{R}$ is normal, that is,

$$
[\varphi, \varphi](X, Y)+d \eta(X, Y) \xi=0
$$

where $[\varphi, \varphi]$ is the Nijenhuis torsion tensor for $\varphi$.

An almost contact metric structure is said to be a contact structure if

$$
\Phi(X, Y)=d \eta(X, Y) \text {. }
$$

A Sasakian manfold is an almost contact metric manifold satisfying (1.6) and (1.7). But it is well known that

$$
\left(\nabla_{X} \varphi\right) Y=-g(X, Y) \xi+\eta(Y) X
$$

is the necessary and sufficient condition for an almost contact metric manifold to be a Sasakian manifold.

An odd dimensional euclidean space $\boldsymbol{R}^{2 n+1}$, a principal circle bundle by Boothby-Wang fibration over a Hodge manifold, a hypersurface of a Kaehler manifold, and a Brieskorn manifold are examples of Sasakian manifolds. (For more detailed theory of contact manifold, see Blair [2])

In a Sasakian manifold we have

$$
\nabla_{X} \xi=\varphi X .
$$


By (1.8), (1.9) and the Ricci identity for $\xi$ we have

$$
R_{k j i}{ }^{h} \xi^{i}=\delta_{k}{ }^{h} \eta_{j}-\delta_{j}{ }^{h} \eta_{k},
$$

or

$$
R_{k j i}{ }^{h} \eta_{h}=\eta_{k} g_{j i}-\eta_{j} g_{k i} .
$$

By applying ${\delta_{h}}^{k}$ to (1.10), we have

$$
R_{j i} \xi^{2}=2 n \eta_{j} .
$$

The Ricci identity for $\varphi$ and (1.8) and (1.9) lead to

$$
R_{k j l}{ }^{h} \varphi_{i}{ }^{l}-R_{k j i}{ }^{l} \varphi_{l}{ }^{h}=-\varphi_{k}{ }^{h} g_{j i}+\varphi_{\jmath}{ }^{h} g_{k i}-\delta_{k}{ }^{h} \varphi_{j i}+\delta_{\jmath}{ }^{h} \varphi_{k l} .
$$

$\delta_{h}{ }^{k}$ applied to (1.13) implies

$$
R_{j l} \varphi_{\imath}{ }^{l}+R_{k j i n} \varphi^{k h}=-(2 n-1) \varphi_{j i},
$$

thus

$$
R_{j l} \varphi_{i}{ }^{l}+R_{i l} \varphi_{\jmath}{ }^{l}=0 .
$$

Furthermore by using the first Bianchi identity, we have

$$
R_{k h j i} \varphi^{k h}=2 R_{j l} \varphi_{i}{ }^{l}+2(2 n-1) \varphi_{j i} .
$$

Considering (1.16) as 2-forms then taking the exterior derivative then using (1.5) and the second Bianchi identity, we have

$$
\begin{aligned}
\nabla_{k} R_{\jmath}-\nabla_{j} R_{k l}=\varphi_{i}{ }^{m} \varphi_{\jmath}{ }^{l} \nabla_{m} R_{k l} & +4 n \varphi_{k \jmath} \eta_{i}-2 n \varphi_{i k} \eta_{\jmath} \\
& -2 R_{\jmath l} \varphi_{k}{ }^{l} \eta_{l}+R_{k l} \varphi_{i}{ }^{l} \eta_{\jmath},
\end{aligned}
$$

and

$$
\nabla_{k} R_{j i}=\varphi_{i}{ }^{m} \varphi_{k}{ }^{l}\left(\nabla_{j} R_{m l}-\nabla_{m} R_{\jmath l}\right)+2 n \varphi_{k \jmath} \gamma_{i}-R_{\jmath l} \varphi_{k}{ }^{l} \eta_{l} .
$$

From (1.18) we easily see that

$$
\left(\nabla_{\xi} \operatorname{Ricci}\right)(\varphi X, \varphi Y)=0 \text {. }
$$

(1.19) suggests the following definition: If the Ricci tensor $R_{j i}$ of a Sasakian manifold $M^{2 n+1}$ satisfies

$$
\left(\nabla_{X} \operatorname{Ricci}\right)(\varphi Y, \varphi Z)=0
$$

for any vector fields $X, Y$ and $Z$ on $M^{2 n+1}$, then the Ricci tensor $R_{j i}$ on $M^{2 n+1}$ is said to be $\eta$-parallel. It is known that if $M^{2 n+1}$ is a regular Sasakian manifold, then $R_{j i}$ on $M^{2 n+1}$ is $\eta$-parallel if and only if the Ricci tensor on $M^{3 n+1} / \xi$ is parallel. (See Kon [5]) From (1.18) we see that in a Sasakian manifold with $\eta$-parallel Ricci tensor we have

$$
\nabla_{k} R_{j i}=2 n\left(\varphi_{k}, \eta_{i}-\varphi_{i k} \eta_{j}\right)-R_{j l} \varphi_{k}{ }^{l} \eta_{i}+R_{k l} \varphi_{i}{ }^{l} \eta_{j}
$$

and thus

$$
\nabla_{k} R_{j i}+\nabla_{j} R_{\imath k}+\nabla_{\imath} R_{k \jmath}=0
$$


We also see from (1.18) that in a Sasakian manifold $M^{2 n+1}$ with $\eta$-parallel Ricci tensor the scalar curvature $S$ is constant and the square of the length of the Ricci tensor is constant. From (1.17) and (1.18) it is easily seen that

$$
\mid \nabla \text { Ricci }\left.\right|^{2}=2 \mid \text { Ricci }\left.\right|^{2}-8 n S+16 n^{3}+8 n^{2}
$$

is the necessary and sufficient condition for a Sasakian manifold to have the $\eta$-parallel Ricci tensor.

Let $M^{2 n+1}$ be a Sasakian manifold. The sectional curvature of the section spanned by $X$ and $\varphi X$ which are orthogonal to $\xi$ is called a $\varphi$-sectional curvature. A Sasakian manifold of constant $\varphi$-sectional curvature $c$ is called a Sasakian space form $M^{2 n+1}(c)$. The necessary and sufficient condition for a Sasakian manifold $M^{2 n+1}(2 n+1 \geqq 5)$ to be a Sasakian space form $M^{2 n+1}(c)$ is that the curvature tensor has the following form:

$$
\begin{aligned}
R(X, Y) Z= & \frac{c+3}{4}(g(Y, Z) X-g(X, Z) Y)+\frac{c-1}{4}(\eta(X) \eta(Z) Y \\
& -\eta(Y) \eta(Z) X+g(X, Z) \eta(Y) \xi-g(Y, Z) \eta(X) \xi \\
& +g(\varphi Y, Z) \varphi X-g(\varphi X, Z) \varphi Y-2 g(\varphi X, Y) \varphi Z) .
\end{aligned}
$$

If the curvature tensor is of the form (1.24), the Ricci tensor $R_{j i}$ and the scalar curvature $S$ are given by

$$
\begin{aligned}
\operatorname{Ricci}(X, Y)= & \frac{n(c+3)+c-1}{2} g(X, Y) \\
& -\frac{(n+1)(c-1)}{2} \eta(X) \eta(Y)
\end{aligned}
$$

and

$$
S=\frac{1}{2}(n(2 n+1)(c+3)+n(c-1)) .
$$

An odd dimensional sphere $S^{2 n+1}$, and odd dimensional euclidian space $\boldsymbol{R}^{2 n+1}$ and the product bundle $\left(\boldsymbol{R}, C D^{n}\right)$, where $C D^{n}$ is a simply connected homogeneous complex domain with constant holomorphic sectional curvature $\leqq 0$ and $\boldsymbol{R}$ is the real line, are examples of Sasakian space forms.

By generalizing (1.25), we call a Sasakian manifold $M^{2 n+1} C$-Einstern if the Ricci tensor $R_{j \imath}$ of $M^{2 n+1}$ is of the form

$$
R_{j i}=a g_{j i}+b \eta_{j} \eta_{\imath}, \text { where } a+b=2 n .
$$

Remark. The second Bianchi identity reduces to

$$
\nabla_{\jmath} S-2 \nabla_{\imath} R_{\jmath}{ }^{\imath}=0 .
$$

From (1.27), the scalar curvature is expressed by

$$
S=(2 n+1) a+b=2 n(a+1) .
$$

By putting (1.27) and (1.29) into (1.28), we have 


$$
(2 n-2) \nabla_{j} a+2 \eta_{j} \xi^{i} \nabla_{i} a=0,
$$

since (1.9) and (1.3) hold. Applying $\xi^{\jmath}$, we see

$$
2 n \xi^{j} \nabla_{j} a=0 \text {. }
$$

Thus if $n>1, a$ and $b$ in (1.27) are necessarily constants, because (1.30) with the second term replaced by (1.31) gives

$$
(2 n-2) \nabla_{j} a=0 .
$$

\section{$\S 2$. Contact Bochner curvature tensor.}

From this section Sasakian manifolds always have dimension $\geqq 5$.

The contact Bochoner curvature tensor $B$ of a Sasakian manifold $M^{2 n+1}$ with structure tensor $(\varphi, \xi, \eta, g)$ is introduced as an analogue of the Weyl conformal curvature tensor of a Riemannian manifold. (See Matsumoto and Chūman [6]) But we do not know as to what kind of non-trivial transformation leaves the contact Bochoner curvature tensor invariant.

where

$$
\begin{aligned}
B_{k j i}{ }^{h}=R_{k j i}{ }^{h} & +\left(\delta_{k}{ }^{h}-\eta_{k} \xi^{h}\right) L_{j \imath}-\left(\delta_{\jmath}{ }^{h}-\eta_{\jmath} \xi^{h}\right) L_{k \imath} \\
& +L_{k}{ }^{h}\left(g_{j i}-\eta_{\jmath} \eta_{\imath}\right)-L_{\jmath}{ }^{h}\left(g_{k i}-\eta_{k} \eta_{\imath}\right) \\
& +\varphi_{k}{ }^{h} M_{j i}-\varphi_{\jmath}{ }^{h} M_{k i}+M_{k}{ }^{h} \varphi_{\jmath i}-M_{\jmath}{ }^{h} \varphi_{k \imath} \\
& -2\left(\varphi_{k j} M_{\imath}{ }^{h}+M_{k j} \varphi_{i}{ }^{h}\right) \\
& +\left(\varphi_{k}{ }^{h} \varphi_{j i}-\varphi_{\jmath}{ }^{h} \varphi_{k i}-2 \varphi_{k j} \varphi_{i}{ }^{h}\right),
\end{aligned}
$$

$$
L_{\jmath i}=\frac{1}{2(n+2)}\left(-R_{\jmath 2}-(L+3) g_{j i}+(L-1) \eta_{\jmath} \eta_{2}\right)
$$

and

$$
\begin{aligned}
& L_{\jmath}{ }^{\prime}=L_{j t} g^{t \imath}, \\
& L=g^{j i} L_{j i}, \\
& M_{\jmath \imath}=-L_{\jmath t} \varphi_{\imath}{ }^{t},
\end{aligned}
$$

From (2.2) and (2.4) it follows that

$$
L=-\frac{S+2(3 n+2)}{4(n+1)},
$$

where $S$ is the scalar curvature of $M^{2 n+1}$.

Applying (1.12) to (2.2), we have

$$
L_{j i} \xi^{2}=-\eta_{j},
$$

which, together with (2.5) yields 


$$
M_{j t} \varphi_{i}{ }^{t}=L_{\jmath i}+\eta_{\jmath} \eta_{\imath} .
$$

The following identities are easily verified:

$$
\begin{aligned}
& B_{k j i}{ }^{h}+B_{j k \imath}{ }^{h}=0, \\
& B_{k j i}{ }^{h}+B_{j i k}{ }^{h}+B_{\imath k \jmath}{ }^{h}=0, \\
& B_{t j i}{ }^{t}=0, \\
& B_{k j i h}+B_{k j h \imath}=0, \\
& B_{k j i h}=B_{i h k \jmath}, \\
& B_{k j i}{ }^{h} \eta_{h}=0, \\
& B_{k j{ }^{h}}{ }^{h} \varphi_{i}{ }^{t}=B_{k j i}{ }^{t} \varphi_{t}{ }^{h}, \\
& B_{k j i}{ }^{h} \varphi^{k \jmath}=0 .
\end{aligned}
$$

Since the vanishing of the Weyl conformal curvature tensor has an important geometric meaning, next, we will study some geometric implications of the vanishing of the contact Bochoner curvature tensor, i. e., $B=0$.

First we have

Proposition 2.1. Let $M^{2 n+1}$ be a Sasakian manifold. If $M^{2 n+1}$ has constant $\varphi$-sectional curvature, then $M^{2 n+1}$ is $C$-Einstein and the contact Bochoner curvature tensor $B$ vanishes.

Proof The first part was already observed in (1.25), so we will just prove the second part. By using (1.25) and (1.26) we have

$$
L=-\frac{n c+3 n+4}{4}
$$

and

$$
L_{\jmath i}=-\frac{c+3}{8} g_{j i}+\frac{c-5}{8} \eta_{\jmath} \eta_{\imath},
$$

thus

$$
M_{j i}=-\frac{c+3}{8} \varphi_{j i}
$$

which, substituted in (2.1), gives the result.

Q.E.D.

The converse of Proposition 2.1 is given in the next proposition.

Proposition 2.2. Let $M^{2 n+1}$ be a Sasakian manfold. If the contact Bochoner curvature tensor vanishes and $M^{2 n+1}$ is a C-Einstein manifold, then $M^{2 n+1}$ has a constant $\varphi$-sectional curvature.

Proof. Since $M^{2 n+1}$ is $C$-Einstein, the Ricci tensor is expressed by $R_{j i}=$ 
$a g_{j i}+b \eta_{j} \eta_{2}$, where $a$ and $b$ are necessarily constants such that $a+b=2 n$. Thus the scalar curvature $S=(2 n+1) a+b$ is constant. By using (3.1), we can compute $R_{k j i}{ }^{h}$, which has the form (1.24) with

$$
c=\frac{2 n a+4 a-3 n^{2}-5 n+2}{(n+1)(n+2)} .
$$

Next, we will weaken our condition on the contact Bochoner curvature tensor and assume that the contact Bochoner curvature tensor is parallel, that is, $\nabla B=0$.

The following proposition gives one sufficient condition for a Sasakıan manifold to have the $\eta$-parallel Ricci tensor.

Proposition 2.3. Let $M^{2 n+1}$ be a Sasakian manifold with parallel conlact Bochoner curvature tensor and constant scalar curvature. Then the Rucc tensor of $M^{2 n+1}$ is $\eta$-parallel.

Proof. By using the curvature properties of a Sasakian manifold that were given in $\S 1$, we obtain the following formula straightforwardly:

$$
\begin{aligned}
\nabla_{h} B_{k j i}{ }^{h}= & -2 n\left(\nabla_{k} L_{j i}-\nabla_{j} L_{\imath k}-\eta_{k}\left(\varphi_{j i}+M_{j i}\right)\right. \\
& -\eta_{j}\left(\varphi_{i k}+M_{\imath k}\right)-2 \eta_{i}\left(\varphi_{j k}+M_{j k}\right) \\
& \left.+\frac{1}{4(n+1)(n+2)}\left(\varphi_{j_{i}} \varphi_{k}{ }^{h}+\varphi_{i_{k}} \varphi_{j}{ }^{h}+2 \varphi_{j k} \varphi_{i}{ }^{h}\right) \nabla_{h} S\right) .
\end{aligned}
$$

By applying $\varphi_{v}{ }^{t} \varphi_{u}{ }^{k} \varphi_{t}{ }^{j} \varphi_{s}{ }^{2}$ and making use of (1.18), we obtain

$$
\nabla_{s} R_{k t} \varphi_{u}{ }^{k} \varphi_{v}{ }^{t}=0 .
$$

Differentiating (2.1) directly, we have

Proposition 2.4. Under the same assumption on $M^{2 n+1}$, the curvature tensor $R$ of $M^{2 n+1}$ satisfies

$$
\left(\nabla_{X} R\right)(\varphi Y, \varphi Z, \varphi V, \varphi W)=0 .
$$

If the curvature tensor $R$ of a Sasakian manifold $M^{2 n+1}$ satisfies the condition (2.19), $M^{2 n+1}$ is said to be a locally D-symmetruc space. It is easy to check that when $M^{2 n+1}$ is a regular Sasakian manifold, $M^{2 n+1}$ is a locally $D$-symmetric space if and only if $M^{2 n+1} / \xi$ is a locally symmetric space since $\xi$ is a Killing vector field.

Now we compute the length of the contact Bochoner curvature tensor of a Sasakian manifold $M^{2 n+1}$ and study some applications.

By computing assiduously, we have

$$
|B|^{2}=|R|^{2}-\frac{8}{n+2} \mid \text { Ricci }\left.\right|^{2}+\frac{2}{(n+1)(n+2)} S^{2}
$$




$$
+\frac{4\left(3 n^{2}+3 n-2\right)}{(n+1)(n+2)} S-\frac{4 n\left(6 n^{3}+9 n^{2}-n-2\right)}{(n+1)(n+2)} .
$$

The same result is obtained independently by D. Janssens [3]. As a preparation we prove the following lemma:

Lemma 2.5. Let $M^{2 n+1}$ be a Sasakıan manifold. Then we have

$$
\mid \text { Ricci }\left.\right|^{2} \geqq \frac{(S-2 n)^{2}}{2 n}+4 n^{2} .
$$

Equality holds if and only if $M^{2 n+1}$ is a C-Einstem manifold.

Proof. At each point $p$ of $M^{2 n+1}$, choose an orthonormal basis including the characteristic vector field $\xi$ of $T_{p} M^{2 n+1}$ so that the matrix representing the Ricci tensor $R_{j \imath}$ is diagonalized. Then the scalar curvature is expressed by

$$
S=\sum_{\imath=1}^{2 n} R_{\imath \imath}+2 n
$$

since $\operatorname{Ricci}(\xi, \xi)=2 n$.

By using Schwartz inequality, we get

$$
(S-2 n)^{2}=\left(\sum_{\imath=1}^{2 n} R_{i i}\right)^{2} \leqq 2 n \sum_{\imath=1}^{2 n} R_{\imath \imath}{ }^{2}=2 n\left(\mid \text { Ricci }\left.\right|^{2}-4 n^{2}\right),
$$

giving the inequality. Equality holds if and only if the Ricci operator restricted to the contact distribution $D$ is $\frac{S-2 n}{2 n} I$, where $I$ denote the identity. This means $R_{j i}=\frac{S-2 n}{2 n} g_{j i}$ for $1 \leqq i, \jmath \leqq 2 n$. Since $\operatorname{Ricci}(\xi, \xi)=2 n$, we have

$$
R_{\jmath i}=\frac{S-2 n}{2 n} g_{j i}+\left(2 n-\frac{S-2 n}{2 n}\right) \eta_{\jmath} \eta_{2} . \quad \text { Q. E. D. }
$$

Now we establish an inequality involving the curvature tensor $R$ and the scalar curvature $S$.

Proposition 2.6. Let $M^{2 n+1}$ be a Sasakzan manifold. Then the following inequality holds

$$
|R|^{2} \geqq \frac{2}{n(n+1)} S^{2}-\frac{4(3 n+1)}{n+1} S+\frac{4 n(3 n+1)(2 n+1)}{n+1} .
$$

Equality holds if and only if $M^{2 n+1}$ has a constant $\varphi$-sectional curvature.

Proof. First we rewrite $|B|^{2}$ so that we can use (2.21).

$$
|B|^{2}=-\frac{8}{n+2}\left(\mid \text { Ricci }\left.\right|^{2}-\frac{(S-2 n)^{2}}{2 n}-4 n^{2}\right)
$$




$$
\begin{aligned}
& +|R|^{2}-\frac{2}{n(n+1)} S^{2}+\frac{4(3 n+1)}{n+1} S \\
& -\frac{4 n(3 n+1)(2 n+1)}{n+1} .
\end{aligned}
$$

Now by virtue of $|B|^{2} \geqq 0$ and (2.21), we have the result.

When the equality holds, $M^{2 n+1}$ is a $C$-Einstein Sasakian manifold with vanishing contact Bochoner curvature tensor. Thus by Proposition $2.2 M^{2 n+1}$ has a constant $\varphi$-sectional curvature. The converse is also true by Proposition 2.1.

Q. E. D.

\section{§. Spectrum of Sasakian manifolds.}

Let $\left(M^{n}, g\right)$ be a compact orientable Riemannian manifold without boundary with a Riemannian metric $g$. The Laplactan $\Delta$, acting on the real valued $C^{\infty}$ function on $M^{n}\left(=C^{\infty}(M)\right)$, is defined by

$$
\Delta f=-\frac{1}{\sqrt{g}} \sum_{i, j=1}^{n} \frac{\partial\left(\sqrt{g} g^{\imath \jmath} \partial f / x^{j}\right)}{\partial x^{\imath}},
$$

where $g=\operatorname{det}\left(g_{\imath \jmath}\right)$ and $\left\{x^{i}\right\}$ is a local coordinate system of $M^{n}$.

The spectrum of $\left(M^{n}, g\right)$, denoted by $\operatorname{Spec}\left(M^{n}, g\right)$, is the set of eigenvalues $\lambda$ of $\Delta$, i. e., the $\lambda$ 's $\in \boldsymbol{R}$ such that there exists $f \in C^{\infty}(M), f \neq 0$ with $\Delta f=\lambda f$. We write

$$
\operatorname{Spec}\left(M^{n}, g\right)=\left\{0=\lambda_{0}<\lambda_{1} \leqq \lambda_{2} \leqq \cdots\right\},
$$

each $\lambda$ being written a number of times equal to its multiplicity, which is known to be finite.

One of the ways to observe the geometric meaning of the spectrum of a manifold is the asymptotic expansion of $\sum_{i} e^{-\lambda_{i} t}$. In particular we have

Theorem 3.1. For every Riemannan manfold, there exist $a_{\imath}$ 's $(\imath=0,1, \cdots)$ with

$$
\sum_{\imath} e^{-\lambda_{\imath} t}=(4 \pi t)^{-n / 2} \sum_{\imath=1}^{k} a_{i} t^{2}+O\left(t^{k+1-n / 2}\right)
$$

for every $k$.

Theoretically we can compute the $a_{\imath}$ 's. But so far only $a_{0}, a_{1}, a_{2}$, and $a_{3}$ have been computed.

$$
\begin{aligned}
a_{0}=\int_{M} v_{g} & =\text { volume of }\left(M^{n}, g\right) \\
a_{1} & =\frac{1}{6} \int_{M} S v_{g},
\end{aligned}
$$




$$
a_{2}=\frac{1}{360} \int_{M}\left(2|R|^{2}-2|\operatorname{Ricci}|^{2}+5 S^{2}\right) v_{g},
$$

and

$$
a_{3}=\frac{1}{6 !} \int_{M} f v_{g},
$$

where

$$
\begin{aligned}
f= & -\frac{142}{63}|\nabla S|^{2}-\frac{26}{63} \mid \nabla \text { Ricci }\left.\right|^{2}-\frac{1}{9}|\nabla R|^{2} \\
& +\frac{5}{9} S^{2}-\frac{2}{3} S \mid \text { Ricci }\left.\right|^{2}+\frac{2}{3} S|R|^{2}-\frac{4}{7} R_{h}{ }^{j} R_{\jmath}{ }^{2} R_{\imath}{ }^{h} \\
& -\frac{20}{63} R^{k \imath} R^{\jmath h} R_{k \jmath \imath}-\frac{8}{63} R^{t s} R_{t}{ }^{j i h} R_{s \jmath i n} \\
& -8{ }_{21} R_{i h}{ }^{m l} R_{m \iota^{k \jmath}} R_{k \jmath}{ }^{i h} .
\end{aligned}
$$

( $a_{3}$ is obtained by Sakai [7], but his curvature tensor has the opposite sign.)

Several results have been obtained by using these $a_{\imath}$ 's by Berger, Sakai, Mckean-Singer, Patodi, Tanno, etc. In particular Tanno [8] obtained

THEOREM 3.2. Let $\left(M^{n}, g\right)$ be a compact orientable Riemannan manfold, $2 \leqq n \leqq 6$. If $\operatorname{Spec}\left(S^{n}, g_{0}\right)=\operatorname{Spec}\left(M^{n}, g\right)$, then $\left(M^{n}, g\right)$ is isometric to $\left(S^{n}, g_{0}\right)$, where $\left(S^{n}, g_{0}\right)$ is an n-dimensional sphere with standard metruc $g_{0}$.

Now we consider the problem of this direction on a Sasakian manifold. First we prove

Proposition 3.3. Let $\left(M^{2 n+1}(c), g\right)$ be a $2 n+1(\geqq 5)$ dimensional compact Sasakian space form of a constant $\varphi$-sectional curvature $c$ and let $\left(M^{*}, g^{*}\right)$ be a compact C-Einstein Sasakran manifold. If $\operatorname{Spec}\left(M^{2 n+1}(c), g\right)=\operatorname{Spec}\left(M^{*}, g^{*}\right)$, then $M^{*}$ is a $(2 n+1)$-dimensional Sasakian space form of a constant $\varphi$-sectional curvature $c^{*}=c$.

Proof. The isospectral condition means the equivalence of $a_{2} s$ and $a_{2}{ }^{*} s$. Thus we have $\operatorname{dim} M^{*}=2 n+1$ and

$$
\begin{aligned}
& \int_{M} v_{g}=\int_{M *} v_{g *}^{*}, \\
& \int_{M} S v_{g}=\int_{M *} S^{*} v_{g *}^{*} .
\end{aligned}
$$

Recall that a $C$-Einstein Sasakian manifold has a constant scalar curvature. Thus from (3.8) and (3.9) we see $S=S^{*} . a_{2}$, in general, is expressed by 


$$
\begin{aligned}
& a_{2}=\frac{1}{360} \int_{M}\left(2|R|^{2}-2|\operatorname{Ricci}|^{2}+5 S^{2}\right) v_{g} \\
&=\frac{1}{360} \int_{M}[ 2\left(|R|^{2}-\frac{2}{n(n+1)} S^{2}+\frac{4(3 n+1)}{n+1} S-\frac{4 n(3 n+1)(2 n+1)}{n+1}\right) \\
& \quad-2\left(|\operatorname{Ricci}|^{2}-\frac{(S-2 n)^{2}}{2 n}-4 n^{2}\right) \\
&\left.+\frac{5 n^{2}+4 n+3}{n(n+1)} S^{2}-\frac{4(5 n+1)}{n+1} S+\frac{4 n(5 n+1)(2 n+1)}{n+1}\right] v_{g} .
\end{aligned}
$$

Thus in our case, the assumption implies that

$$
\begin{aligned}
& \frac{5 n^{2}+4 n+3}{n(n+1)} \int_{M} S^{2} v_{g} \\
& =\int_{M *} 2\left(\left|R^{*}\right|^{2}-\frac{2}{n(n+1)} S^{* 2}+\frac{4(3 n+1)}{n+1} S^{*}\right. \\
& \left.\quad+\frac{4 n(3 n+1)(2 n+1)}{n+1}\right) v^{* *} \\
& +\frac{5 n^{2}+4 n+3}{n(n+1)} \int_{M *} S^{* 2} v^{*}{ }^{*} .
\end{aligned}
$$

Since both $S$ and $S^{*}$ are constants and $S=S^{*}$, we have the second line of (3.11) vanishes. Proposition 2.6 shows that $M^{*}$ is a Sasakian space form. By (1.26) $S=S^{*}$ implies $c=c^{*}$.

Q.E.D.

We can improve Proposition 3.3 in the following sense by using the contact Bochoner curvature tensor.

TheOREM 3.4. Let $\left(M^{2 n+1}(c), g\right)$ be a compact Sasakian space form of a constant $\varphi$-sectional curvature $c$ of dimension $2 n+1=5,7,9$ or 11 , and let $\left(M^{*}, g^{*}\right)$ be a compact Sasakian manifold. If $\operatorname{Spec}\left(M^{2 n+1}(c), g\right)=\operatorname{Spec}\left(M^{*}, g^{*}\right)$, then $M^{*}$ is a Sasakian space form of a constant $\varphi$-sectronal curvature $c^{*}=c$ of the same dimension as that of $M^{2 n+1}(c)$.

Proof. From the assumption we have

$$
\begin{array}{r}
\operatorname{dim} M^{*}=2 n+1, \\
\int_{M} v_{g}=\int_{M *} v^{* *}, \\
\int_{M} S v_{g}=\int_{M *} S^{*} v^{*}{ }_{g *} .
\end{array}
$$

By using the length of the contact Bochoner curvature tensor, $a_{2}$, in general, is expressed by

$$
a_{2}=\frac{1}{360} \int_{M}\left[2|B|^{2}+\frac{2(6-n)}{n+2}\left(|\operatorname{Ricci}|^{2}-\frac{(S-2 n)^{2}}{2 n}-4 n^{2}\right)\right.
$$




$$
\left.+\frac{5 n^{2}+4 n+3}{n(n+1)} S^{2}-\frac{4(5 n+1)}{n+1} S+\frac{4 n(5 n+1)(2 n+1)}{n+1}\right] v_{g} .
$$

Now our assumption $a_{2}=a_{2}{ }^{*}$ together with other assumption implies that

$$
\begin{aligned}
& \frac{5 n^{2}+4 n+3}{n(n+1)} S^{2} \int_{M} v_{g} \\
&=\int_{M *}\left[2\left|B^{*}\right|^{2}+\frac{2(6-n)}{n+2}\left(\mid \text { Ricci* }^{*}-\frac{\left(S^{*}-2 n\right)^{2}}{2 n}-4 n^{2}\right)\right] v^{*}{ }_{g^{*}} \\
&+\frac{5 n^{2}+4 n+3}{n(n+1)} \int_{M *} S^{*} v^{*}{ }_{g *} .
\end{aligned}
$$

Because of Lemma 2.5, we see that

$$
S^{2} \int_{M} v_{g} \geqq \int_{M *} S^{* 2} v^{*}{ }_{g *}
$$

On the other hand using (3.12), (3.13) and the Schwartz's inequality, we have

$$
\begin{aligned}
& S^{2} \int_{M} v_{g} \int_{M *} v^{*}{ }_{g}^{*} \\
& \quad=S^{2}\left(\int_{M} v_{g}\right)^{2}=\left(\int_{M} S v_{g}\right)^{2}=\left(\int_{M *} S^{*} v^{*}{ }_{g *}\right)^{2} \\
& \quad \leqq \int_{M *} S^{* 2} v^{*} \int_{g^{*}} v^{*}{ }_{g^{*}},
\end{aligned}
$$

that is,

$$
S^{2} \int_{M} v_{g} \leqq \int_{M *} S^{* 2} v^{*}{ }_{g *}
$$

Hence equality holds in (3.17), which means $S^{*}=S=$ constant.

Looking back (3.15) we see that $B^{*}=0$ and $\left|\operatorname{Ricci}^{*}\right|^{2}=\frac{\left(S^{*}-2 n\right)^{2}}{2 n}+4 n^{2}$ in our situation. Lemma 2.5 and Proposition 2.2 imply that $M^{*}$ is a Sasakian space form, whose constant $\varphi$-sectional curvature $c^{*}=c$ because of $S^{*}=S$. Q. E. D.

The expression (3.14) gives the following corollary:

Corollary 3.5. Let $(M, g)$ and $\left(M^{*}, g^{*}\right)$ be compact Sasakian manrfolds with $\operatorname{dim} M=13$. If $\operatorname{Spec}(M, g)=\operatorname{Spec}\left(M^{*}, g^{*}\right)$, then the contact Bochoner curvature tensor $B$ of $M$ vanishes and the scalar curvature $S$ of $M$ is constant, if and only if the contact Bochoner curvature tensor $B^{*}$ of $M^{*}$ vanishes and the scalar curvature $S^{*}$ of $M^{*}$ is constant.

In order to extend Theorem 3.4 to 13-dimensional case, we shall use $a_{3}$ in (3.6) and (3.7). Since we have Corollary 3.5, we only have to consider the case 
of $B=0$ and $S=$ constant. After simplifying the expression of $a_{3}$ term by term under this assumption we have

LEMMA 3.6. Let $\left(M^{2 n+1}, g\right)$ be a compact Sasakran manufold of dimension $2 n+1$ ( $\geqq 5)$. If the contact Bochoner curvature tensor $B$ of $M^{2 n+1}$ vanishes and the scalar curvature $S$ of $M^{2 n+1}$ is constant, then $a_{3}$ of $M^{2 n+1}$ is expressed by

$$
\begin{array}{r}
a_{3}=\frac{1}{6 !} \int_{M}\left[\frac{2(A(n) S-B(n))}{63 n(n+1)(n+2)^{2}}\left(\mid \text { Ricci }\left.\right|^{2}-\frac{(S-2 n)^{2}}{2 n}-4 n^{2}\right)\right. \\
\left.+C_{1}(n) S^{3}+C_{2}(n) S^{2}+C_{3}(n) S+C_{4}(n)\right] v_{g},
\end{array}
$$

where

$$
\begin{aligned}
& A(n)=-21 n^{5}+21 n^{4}+454 n^{3}+936 n^{2}+776 n+240, \\
& B(n)=4 n\left(61 n^{3}+435 n^{2}+788 n+420\right),
\end{aligned}
$$

and the $C_{k}(n)^{\prime} s, k=1,2,3,4$ are the algebraic expressions in $n .^{(*)}$

THEOREM 3.7. Let $\left(M^{13}(c), g\right)$ be a 13-dimensional Sasakian space form of a constant $\varphi$-sectional curvature $c \neq 31$, and let $\left(M^{*}, g^{*}\right)$ be a compact Sasakian manifold. If $\operatorname{Spec}\left(M^{13}(c), g\right)=\operatorname{Spec}\left(M^{*}, g^{*}\right)$, then $M^{*}$ is a 13-dimensional Sasakian space form of a constant $\varphi$-sectional curvature $c^{*}=c$.

Proof. First we have $\operatorname{dim} M^{*}=13$. Next, by Proposition 2.1 and Corollary 3.5 , the contact Bochoner curvature tensor $B^{*}$ of $M^{*}$ vanishes and the scalar curvature $S^{*}$ of $M^{*}$ is constant. Our assumption $a_{0}=a_{0}^{*}$ and $a_{1}=a_{1}^{*}$ imply that the scalar curvature $S$ and $S^{*}$ of $M^{13}(c)$ and $M^{*}$ respectively are equivalent. From (1.26) we see that $c \neq 31$ means $S=S^{*} \neq 1416$.

Now we only have to prove that $M^{*}$ is a $C$-Einstein manifold. (3.18) with $n=6$ is given by

$$
\begin{array}{r}
a_{3}=\frac{1}{6 !} \int_{M}\left[\frac{576 S-815616}{169344}\left(\mid \text { Ricci }\left.\right|^{2}-\frac{(S-12)^{2}}{12}-144\right)\right. \\
\left.+C_{1}(6) S^{3}+C_{2}(6) S^{2}+C_{3}(6) S+C_{4}(6)\right] v_{g} .
\end{array}
$$

The assumption $a_{3}=a_{3}{ }^{*}$, together with other assumptions, implies

$$
0=\int_{M *}\left[\frac{576 S^{*}-815616}{169344}\left(\left|\operatorname{Ricci}^{*}\right|^{2}-\frac{\left(S^{*}-12\right)^{2}}{12}-144\right)\right] v^{*}{ }_{g *}
$$

Since $S^{*} \neq 1416, \mid$ Ricci $\left.^{*}\right|^{2}-\frac{\left(S^{*}-12\right)^{2}}{12}-144=0$. Hence $M^{*}$ is a $C$-Einstein manifold.

Therefore, $M^{*}$ is a Sasakian space form of a constant $\varphi$-sectional curvature

(*) The author would like to thank Professor G. Ch-ūman for pointing out a mistake in the original version of (3.18). 
$c^{*} \neq 31$.

Q.E.D.

An odd-dimensional sphere $S^{2 n+1}$ with the standard metric $g_{0}$ is a compact Sasakian space form of a constant $\varphi$-sectional curvature $c=1$. Here we give a partial answer to one of the questions proposed in the Introduction.

THEOREM 3.8. $\operatorname{Spec}\left(S^{n}, g_{0}\right)=\operatorname{Spec}(M, g)$, under the assumption that $n=5,7$, 9,11 or 13 and that $(M, g)$ is a compact Sasakian manfold, implies that $(M, g)$ is isometric to $\left(S^{n}, g_{0}\right)$.

Proof. Theorem 3.4 and 3.7 imply that $(M, g)$ is a Sasakian space form of a constant $\varphi$-sectional curvature $=1$, that is, a space form of a constant curvature $=1$, with the same volume as that of $\left(S^{n}, g_{0}\right)$. Therefore $(M, g)$ is isometric to $\left(S^{n}, g_{0}\right)$.

Q.E. D.

\section{$\S 4$. Spectrum of 1-forms of Sasakian manifolds.}

By considering the action of the Laplacian $\Delta$ on $p$-forms on a compact orientable Riemannian manifold $\left(M^{n}, g\right)$, we can consider spectrum of $p$-forms:

$$
\operatorname{Spec}^{p}\left(M^{n}, g\right)=\left\{0=\lambda_{0, p}<\lambda_{1, p} \leqq \lambda_{2, p} \leqq \cdots\right\} .
$$

It is again an interesting problem to investigate how the spectra $\left\{\lambda_{2, \gamma}\right\}$ reflect the geometry of $M^{n}$.

The asymptotic expansion in this case is

$$
\sum_{\imath} e^{-\lambda_{\imath, p} t}=(4 \pi t)^{-n / 2} \sum_{\imath=1}^{k} a_{\imath, p} t+O\left(t^{k+1-n / 2}\right) .
$$

The following coefficients are known:

$$
\begin{gathered}
a_{0,1}=n \int_{M} v_{g}=n \text { volume of }\left(M^{n}, g\right), \\
a_{1,1}=\frac{n-6}{6} \int_{M} S v_{g}, \\
a_{2,1}=\frac{1}{360} \int_{M}\left[2(n-15)|R|^{2}+2(90-n)|\operatorname{Ricci}|^{2}+5(n-12) S^{2}\right] v_{g} .
\end{gathered}
$$

By using the similar technique, we get the following results :

Theorem 4.1. Let $(M, g)$ and $\left(M^{*}, g^{*}\right)$ be compact Sasakran manifolds. Assume $\operatorname{Spec}^{1}(M, g)=\operatorname{Spec}^{1}\left(M^{*}, g^{*}\right)$, then we have

(1) $\operatorname{dim} M=\operatorname{dim} M^{*}$,

(2) for $\operatorname{dim} M=\operatorname{dim} M^{*}=17,19,26, \cdots, 101,103, M$ is of a constant $\varphi$-sectional curvature $c$, if and only if $M^{*}$ is of a constant $\varphi$-sectional curvature $c^{*}=c$. 
COROLlARY 4.2. $\operatorname{Spec}^{1}\left(S^{n}, g_{0}\right)=\operatorname{Spec}^{1}(M, g)$, under the assumption that $n=$ $17,19,21, \cdots, 101,103$ and that $(M, g)$ is a compact Sasakian manifold, implies that $(M, g)$ is isometric to $\left(S^{n}, g_{0}\right)$.

Remark. Tanno [9] proved that $\operatorname{Spec}^{1}\left(S^{n}, g_{0}\right)=\operatorname{Spec}(M, g)$ implies that $(M, g)$ is isometric to $\left(S^{n}, g_{0}\right)$ for $n=2,3$ or $16,17,18, \cdots, 92,93$. Hence our corollary applies for $n=95,97,99,101$ and 103 .

\section{BIBLIOGRAPHY}

[1] M. Berger, P. Gauducion and E. Mazet, Le spectre d'une variétés riemannienne, Lecture Notes in Math., Vol. 194, Springer-Verlag, Berlin and New York, 1971.

[2] D. E. Blair, Contact Manifolds in Riemannian Geometry, Lecture Notes in Math., Vol. 509, Springer-Verlag, Berlin and New York, 1976.

[3] D. Janssens, Krommingstensoren Decompositietheoric en Toepassingen, Doctoraatsverhandeling, Katholieke Universiteit Leuven, 1977.

[4] M. KAC, Can one hear the shape of a drum?, Amer. Math. Monthly 73 (1966), no. 4, Part II, 1-23.

[5] M. Kon, Invariant submanifolds in Sasakıan manifolds, Math. Ann. 219 (1976), 277-290.

[6] M. Matsumoto and G. Cheman, On the $C$-Bochoner curvature tensor, TRU Math. 5 (1969), 21-30.

[7] T. SAKAI, On eigenvalues of Laplacian and curvatıre of Riemannian manifold, Tôhoku Math. J. (2) 23 (1971), 589-603.

[8] S. TANNo, Eigenvalues of the Laplacian of Riemannian Manifolds, Tôhoku Math. J. (2) 25 (1973), 391-403.

[9] S. Tanno, The spectrum of the Laplacian for 1-forms, Prco. Amer. Math. Soc. 45 (1974), 125-129.

Department of Mathematics

Michigan State University

EAst Lansing, Michigan, 48824

Department of Mathematics

TOKyo Institute of Technology

Oh-okayama, Meguro, 152, Tokyo, Japan 